\title{
Geoinformation space monitoring in the task of sustainable development of the regions located along the main ground routes
}

\author{
I.G. Zhurkin, A.M. Portnov, and S.S. Druchinin* \\ Moscow State University of Geodesy and Cartography (MIIGAiK), Gorokhovsky lane, 4, 105064 \\ Moscow, Russia
}

\begin{abstract}
The article proposes a concept for assessing the natural resource potential and the ecological state of the territories located along the main land routes in order to ensure the sustainable development of the territories. The relevance of building a geoinformation monitoring system for a comprehensive ecological and economic assessment of the regions located along the main land routes is considered. The existing scientific publications on the assessment of the ecological state of the territories are analyzed, including the mathematical apparatus for the assessing. The author's methodology for the implementation of geoinformation modeling and analysis of remote sensing data using the methods of validation of space information is presented. The set-theoretic models of geoinformation mapping of the natural resource potential of the regions located along the main land routes are presented. The conclusion is made about the advantages of using the methods of geoinformation space monitoring for continuous observation and assessment of the state of the territories located along the main land routes.
\end{abstract}

\section{Introduction}

In connection with the growing need for the rational use of natural resources and ensuring the sustainable development of the territorial entities, there is a need to obtain economic assessments of the natural resource potential (hereinafter - the NRP), the effectiveness of which is especially manifested at the regional level. At the same time, under the economic assessment of the natural resource potential of the region, as a rule, it was accepted "the definition of their (natural resources) value in monetary terms in the fixed socio-economic conditions of production under the specified regimes of nature management and environmental restrictions on economic or other activities" [1]. Currently, there is a steady tendency towards the concentration of labor and financial resources along the main land routes (hereinafter - MLR). The territories adjacent to the MLR are a key place for the development of the urbanization processes (large cities, at the same time, become the transport hubs). In addition to a significant role in the socio-economic sphere, MLRs have a negative impact on the environment, being one of the largest sources of pollution of the

* Corresponding author: druchinin@miigaik.ru 
atmosphere (in the form of exhaust gases) and soil (heavy metals, salinization, chemicals, etc.), noise impact, violations of the migration routes of animals, etc. Naturally, the combination of these factors negatively affects the environmental situation of the regions through which the MLR pass, which, in turn, affects both the market valuation of land and real estate, and the taxation processes, i.e. on the economic assessment of the region's NRP. Thus, only a comprehensive environmental and economic analysis can assess the efficiency of MLR operation for each specific region through which MLR passes. Based on the foregoing, an integral quantitative criterion for the effectiveness of NRP could become an objective indicator for assessing the impact of MLR on the sustainable development of the region, used by the executive authorities, local self-government, when drawing up the territorial planning documents, environmental action plans, etc. understanding of the impact of MLR on the general situation in the region. In view of the fact that the construction and operation of such large, financially costly objects as MLR are designed for long periods, the process of geoinformation aerospace monitoring, associated with the collection and processing of spatial data on the current situation on the route, is of great importance. However, there are virtually no publications in scientific journals on this issue.

\section{Materials and methods}

As mentioned above, it is customary to rely on the NRP for the selected region as a whole, while the methodology for calculating the NRP allows you to perform this task for any region. The authors of this article propose to calculate the NRP taking into account the impact from the construction and operation of MLR passing through this region. To carry out an objective assessment of the development and forecast the development of the region in order to ensure its sustainable development, the question arises of choosing an objective quantitative criterion for assessing the NRP at the stages of design and operation of MLR.

As a basis for such a criterion, it is proposed to take the approach proposed in [2] to assess the ecological state of the territories. In [2], a formula is introduced to determine the integral criterion for assessing the ecological state of the territory (hereinafter - EST) for time $\mathrm{j}$ as follows:

$$
Q_{i}=\left\{\prod_{i=1}^{k} \eta_{i j}^{P i}\right\}_{\Omega},
$$

Where:

$k$ is the number of environmental factors characterizing EST of the territory $\Omega$ at the time $j$; $\eta_{i j}$ - this is the value of the partial criterion of the $i$-th environmental factor for time $j$;

$P_{i}$ - coefficient of the importance of the factor, the numerical value of which is located within the numerical segment $[0,1]$.

Thus, the result of calculating the integral criterion for the EST of the territory depends on the choice of environmental factors and on the obtained values of the partial criterion for assessing each environmental factor; however, the following conditions must be met:

- the values of the criteria should represent a dimensionless quantity;

- the values of the criteria must be determined on the same numerical set;

- the values of the criteria must have the same monotonous character.

In order to fulfill these conditions, the following formula was proposed for assessing the $i$-th environmental factor using a partial criterion:

$$
\eta_{i}=\frac{A_{\Pi i}-A_{\Phi i}}{A_{\Pi i}+A_{\Phi i}},
$$


Where:

$\mathrm{A}_{\Pi i}$ - the limiting value of the $i$-th environmental factor in the territory chosen for the assessment, the excess of which is not allowed;

$\mathrm{A}_{\Phi i}$ - the actual value of the $i$-th environmental factor in the area selected for the assessment.

In this case, it is necessary to observe the conditions under which the numerical values of $\mathrm{A}_{\Pi i}$ and $\mathrm{A}_{\Phi i}$ are positive. Thus, if the numerical value of the partial criterion for assessing the ecological factor $\eta_{i}$ is within the half-interval (0.1], this indicates a favorable influence of the ecological factor on the ecological situation in the selected area. If $\eta_{i}=0$, this means that the influence of the $i$-th environmental factor on the territory of $\Omega$ becomes unacceptable.

Based on the foregoing, it is easy to conclude that in the case when the integral assessment criterion $Q$ takes the value $Q=1$ as a result of calculations, this is an indicator of the most favorable (ideal) ecological situation; if $Q=0$, this indicates an unacceptable (catastrophic) environmental situation.

An identical mathematical method (but with different factors) is also used for the integral assessment of the region's PDP. For example, measured values and maximum permissible concentrations (MPC) of harmful substances in the atmosphere, hydrosphere, pedosphere, etc. established by regulatory documents can be used as partial criteria for assessing TSP. In the integral assessment of the NRP, the aggregates of various economic indicators are taken as partial criteria, for example, the aggregate of costs for the development of minerals and profits from their sale.

As mentioned above, an objective assessment of the state of the region requires an integrated ecological and economic approach. The authors of this article propose to use the following expression as an objective quantitative criterion for a comprehensive ecological and economic assessment of a region located along the MLR:

$$
Q_{\text {region }}=Q_{N R P}^{P i} * Q_{E S T}^{P j} \text {, }
$$

Where:

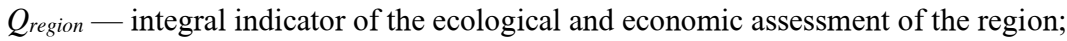

$Q_{N R P}$ - integral indicator for assessing the region's NRP;

$Q_{E S T}$ - integral indicator for assessing the region's EST;

$P_{i}, P_{j}$ - weight coefficients that determine the degree of importance of economic and environmental indicators, respectively (assigned by experts depending on the priority of economic development or environmental safety for a given region located along the MLR).

Since the MLR have a significant length and pass through the territory of several regions, for an integral ecological and economic assessment of the MLR impact on all regions, through which it is planned to lay or has already been laid and operated the route, in accordance with the above approach, one can go to the following expression:

$$
Q_{M L R}=\left\{\prod_{i=1}^{k} Q_{i j \text { region }}\right\}_{\Omega},
$$

Where:

$k$ - number of regions, the aggregate of which forms the territory $\Omega$ on which the MLR is located;

$Q_{i j}$ region - the value of the integral indicator of the ecological and economic assessment of the $i$-th region, located along the MLR, for a time $j$.

For the sustainable development of the region located along the MLR, ensuring its environmental safety, the authors of this article propose the following mathematical expression: 


$$
\begin{aligned}
& Q_{N R P} \rightarrow \max , \\
& Q_{E S T} \leq Q_{\text {threshold }}
\end{aligned}
$$

where:

$$
Q_{N R P} \rightarrow \max -\text { target function; }
$$

$Q_{\text {threshold }}$ - threshold value of the ecological safety of the region.

The main advantage of the above approach is the possibility of using linear programming methods to solve optimization problems to ensure sustainable development of the region located along the MLR, which is ensured by using a quantitative multiplicative criterion for the integral assessment of PNP and EST. For example, taking the logarithm of expression (1) for each region, we get a system of linear equations of the following form:

$$
\ln Q_{i}=\sum_{i=1}^{k} P_{i} * \ln \eta_{i j}
$$

Among the scientific publications devoted to the issues of an integral assessment of the state of the natural environment, published over the past decade, it is worth noting the works of V.V. Dmitriev and members of the research team of St. Petersburg State University [3-8]. In these works, a methodology for calculating the integral indicator is proposed: selection of assessment criteria and selection of classes of the state of natural objects; transformation, on the basis of the analytical desirability function, of the initial characteristics into dimensionless quantities, the set of values of which is located within the numerical interval $[0,1]$; determination of the type of integral indicator, introduction of values of weight coefficients; calculation of the scale of the integral indicator by classes of state; determination of the value of the integral indicator of the state of the system under study at a certain point in time according to the data of field surveys and laboratory studies [3].

In [5], the authors carried out a detailed analysis of various interpretations of the term "system stability", made a study of the advantages and disadvantages of existing methods for assessing the stability of the state of systems (point-index approach, indicator approach, etc.), critically examines the prospect of entering the so-called "universal" index of the state and sustainability of the ecosystem, based on the Shannon index for measuring the amount of ecosystem information (except for the cases of its use for assessing species diversity in the ecosystem), the prospect of using a multi-criteria assessment, namely the method of analysis and synthesis with information deficit (ASID), is approved originally used in socio-economic studies, assessments of urban engineering infrastructure.

Papers [6-8] are of a purely applied nature: they provide the results of assessing the sustainability of various ecosystems in the North-West of Russia based on the following methodology: criteria and parameters of the assessment are determined; a linear convolution of indicators is carried out in order to obtain the dimensionless values; the classes of ecological well-being are distinguished on the numerical interval $[0,1]$, on the basis of which an assessment scale is drawn up, in which the quantitative values of the assessment criteria correlate with the degree of ecological well-being.

Also, from publications on the subject of geomodelling of NRP, published in recent years, one can indicate the works [9-11]. These works are devoted to the practical aspects of the software implementation of the NRP assessment technology. They note the importance of NRP modeling, the need to integrate the arrays of spatial and attributive data for the development of a regional NRP model, demonstrate an extremely generalized structural diagram of the database for determining NRP, provide partial information about the mathematical apparatus for calculating the integral indicator of the state of complex 
natural and artificial systems, and also separate, disparate software solutions in this area. However, these works lack information on the methodological foundations for assessing the natural resource potential of the regions, detailed information on the architecture of building a geographic information system for spatial analysis and digital mapping of natural resource potential, as well as data processing algorithms for geoinformation modeling.

\section{Results and discussion}

To study the state of the ecological systems in the regions and to model the natural resource potential of collection, processing and geoinformation analysis of remote sensing data obtained using multi- and hyperspectral imaging systems. As a result of these actions, it is planned to obtain such data on the state of the ecosystems as: determination of the species composition of herbaceous and forest vegetation, as well as the dynamics of changes in the species composition; the level of development/degradation of various types of vegetation based on the analysis of indicators of vegetation indices [12]; moisture and mineral composition of soils; determination of the boundaries of areas exposed to various pollutants [13]; development of erosion processes, etc. The significant length of MLR necessitate to use space imagery data, which has a significant territorial coverage and the consistency of imagery by remote sensing satellites. At the same time, the need to monitor the state of MLR requires the use of data obtained from unmanned aircraft systems (UAS), which provide a large-scale survey. Thus, the problem arises of bringing the remote sensing data obtained with the use of various imaging systems, both into a single topogeodetic and a single photometric coordinate system. This problem is solved by using the method of complex validation of aerial and space imagery using mobile ground measuring systems [14], including a photogrammetric mark with a calculated center of the original design and a sensitometric wedge, which together represent a mobile ground measuring complex suitable for rapid deployment. in the study area. The validation technology described in [14] makes it possible to simultaneously evaluate the linear resolution of imaging systems and the photometric characteristics of aerial and satellite images by processing the remote sensing data in a specialized software package. The validation of imaging systems is necessary to assess the quality of remote sensing data in order to comply with the accuracy characteristics of the final materials for geoinformation mapping of terrain objects, processes and phenomena occurring within the study area. In turn, the photometric characteristics of the images of the earth's surface have a nonlinear distribution in the images (in particular, the optical density), which leads to the impossibility of effectively using the algorithms for automated decoding, since spectral brightness indicators of terrain objects change their character depending on many factors (for example: weather conditions, season, etc. The use of a mobile ground-based measuring complex simultaneously with aerial and space surveys will allow to overcome this problem.

\section{Conclusion}

The use of the methods and technologies proposed by the authors for geoinformation space monitoring of regions located along the main ground routes has the following advantages:

- The use of a quantitative multiplicative criterion for the integral assessment of the NRP and EST makes it possible to solve the optimization problems of ensuring the sustainable development of regions located along the MLR, due to the possibility of using linear programming methods.

- The large length of MLR requires the use of data from space imagery, which has a significant coverage of the territory. 
- Consistency and continuity of observations is ensured through the use of space survey data.

- In order to ensure monitoring of the MLR state, remote sensing data are required on a large scale survey, which is achieved through the use of unmanned aircraft systems (UAS). It is ensured that aerial and space imagery data is brought into a single photometric coordinate system, thanks to the use of the space information validation complex, which allows processing remote sensing data obtained by various imaging systems.

- Geospatial referencing of aerial and space survey data is provided in a single coordinate system.

\section{Acknowledgment}

The research was carried out within the framework of state task 0708-2020-0001 of the Ministry of science and higher education of the Russian Federation.

\section{References}

1. N.N. Lukyanchikov, Economic and organizational mechanism of environmental and natural resources management, 236 (1998)

2. I.G. Zhurkin, News of higher educational institutions, Geodesy and aerial photography, 2, 105 (1992)

3. V.V. Dmitriev, Society. Environment. Development (Terra Humana), 4 (2009)

4. V.V. Dmitriev, Biosphere., 4 (2010)

5. V.V. Dmitriev, A. H. Ogurtsov, Bulletin of St. Petersburg State University, Earth Sciences, 3 (2012)

6. V.V. Dmitriev, A. H. Ogurtsov, Bulletin of St. Petersburg State University, Earth Sciences, 3 (2013)

7. V.V. Dmitriev, A. H. Ogurtsov, Bulletin of St. Petersburg State University, Earth Sciences, 4 (2014)

8. V. V. Dmitriev, I. V. Fedorova, A. C. Biryukova, Bulletin of St. Petersburg State University, Earth Sciences, 2 (2016)

9. O.N. Nikolaeva, Bulletin of Siberian State University of Geosystems and Technologies, 1(33) (2016)

10. V.A. Rybak, Shokr Ahmad, System Analysis and Applied Informatics, 3 (2017)

11. G.P. Glazunov, N.V. Afonchenko, A.I. Sanzharov, Bulletin of the Kursk State Agricultural Academy, 8 (2017)

12. T.A. Adamovich, G.Ya. Kantor, T.Ya. Ashikhmina, V.P. Savinykh, Theoretical and Applied Ecology, 1, 18 (2018)

13. T.I. Kutyavina, V.V. Rutman, T.Ya. Ashikhmina, V.P. Savinykh, Theoretical and Applied Ecology, 3, 28 (2019)

14. I.G. Zhurkin, G.G. Sychev, V.S. Gruzinov, Proceedings of universities, Geodesy and aerial photography, 3, 81 (2012) 